\title{
Measurement of Relative Position of Camera and Optical Beacon by Simultaneous Passive Method
}

\author{
Jiri Nemecek, and Martin Polasek
}

\begin{abstract}
The paper deals with a problem of a passive measurement of the relative position of an optical beacon and an optical camera by a simultaneous analytical method. The beacon is composed of nine light sources which are arranged in space in a defined way. The proposed beacon layout enables the measurement of the beacon range and one position angle of the camera. This paper presents the mathematical model of the measurement method and the results, which were gathered on the basis of two experimental measurements. The first experiment was only indicative. The extreme results of the second experiment were as follows: the minimum and maximum absolute percentage errors of the beacon range were zero and $1.72 \%$, the minimum and maximum errors of the position angle were $0.1^{\circ}$ and $1.64^{\circ}$. The standard commercial cameras and lenses with different focal lengths were used.
\end{abstract}

Keywords-Lens focal length, measurement, optical beacon, optical camera, relative position, simultaneous passive method.

\section{INTRODUCTION}

Passive methods using images of some objects of interest constitute one group of the methods of measuring the relative position of the objects. Some analytical methods are theoretically developed, experimentally verified, and practically used as well [1]-[5]. There are some researches which use stereo vision for measuring position [6], [7] or combination of one camera and other sensor like a sonar or laser range finder [8], [9]. This task can also be solved using neural networks [10], [11].

The passive method, which is presented in [5], enables to measure the object distance and one directional angle by a monocular vision. The image of the object is used to the separated measurement of the individual coordinates. Our solution is also based on the utilization of one camera but we use the specified object and the simultaneous measurement of individual quantities. This method potentially provides the possibility of measuring three positional coordinates.

This paper deals with the problem of the passive measurement of the position of the optical camera (hereinafter camera) relative to the artificially created object, the optical beacon (hereinafter beacon), with a defined spatial arrangement. The paper presents analytical relations that allow indirect measurement of the required parameters.

For experimental purposes, the beacon was made. Its design is shown in Fig. 1 and Fig. 2. It allows a satisfactory

Manuscript received October 31, 2018; revised January 8, 2019

This work was supported by the Czech Republic Ministry of Defence University of Defence development program "Research of sensor and contro systems to achieve battlefield information superiority".

Jiri Nemecek (corresponding author) and Martin Polasek are with Department of Aircraft Technology, University of Defence in Brno, Brno, Czechia, phone +420 973445 198; e-mail: (jiri.nemecek, martin.polasek)@unob.cz. measurement of the distance between the camera and the beacon (hereinafter beacon range) and the position angle of the camera in the horizontal plane (hereinafter camera azimuth). Nine semiconductor LED sources (hereinafter diode) are used as the sources of optical signals sensed by the camera Depending on the relative position of the diodes on the beacon and the relative position of their images in the plane of the camera sensor, the values of the measured variables are determined. The presented layout of the beacon allows the use of the ratio method of the azimuth measurement.

This article is extended version of the paper Measurement of Relative Position of Camera and Optical Beacon by Simultaneous Passive Method, which was presented at the 41st International Conference on Telecommunications and Signal Processing [12].

\section{Mathematical Measurement Models}

The beacon diodes delineate three walls, one front and two side. All of them are shaped like squares of the same size. The sides of these squares represent the base of the beacon $b(\mathrm{~m})$, see Fig. 1 The $S_{1}$ diode is a referential diode. The horizontal plane $\rho_{\mathrm{h}}$ passes through the beacon centre $C_{\mathrm{B}}$ located in the middle of the front wall $A_{\mathrm{f}}$. The plane of the beacon symmetry $\rho_{\mathrm{v}}$ passes through diode $\mathrm{S}_{1}$ and the beacon centre $C_{\mathrm{B}}$. It is perpendicular to plane $\rho_{\mathrm{h}}$. The front wall and the individual side walls form the beacon opening angle $\beta\left(^{\circ}\right)$. In Fig. 1 areas $A_{\text {slp }}$ and $A_{\text {srp }}$ are orthographic projections of the side walls $A_{\mathrm{sl}}$ and $A_{\mathrm{sr}}$ onto the plane in which the wall $A_{\mathrm{f}}$ lies. The angle $\alpha_{1} 1\left({ }^{\$}\right.$ circ $)$ and distances $d_{16}, d_{12}(\mathrm{~m})$ are as follows:

$$
\begin{gathered}
\alpha_{1}=\arctan \left(\frac{\cos \beta}{0.5+\sin \beta}\right), \\
d_{16}=b \cdot \sqrt{1.25+\sin \beta}, \\
d_{12}=0.5 \cdot b,
\end{gathered}
$$

where $d_{16}, d_{12}$ are the distances between diodes $\mathrm{S}_{1}, \mathrm{~S}_{6}$ and $\mathrm{S}_{1}$, $\mathrm{S}_{2}$, respectively, $\alpha_{1}$ is the angle formed by the line connecting the $S_{1}$ and $S_{6}$ diodes and the plane where the front wall lies, see Fig. 1. The angle $\beta$ and the distance $d_{16}$ are the basic beacon parameters.

If the beacon is desired to be symmetrical for simplicity, the following conditions must be met: $d_{81}=d_{16}, \beta_{1}=\beta_{\mathrm{r}}=\beta$. The parameter $d_{81}$ is the distance between the diodes $S_{8}$ and $\mathrm{S}_{1}$. The parameters $\beta_{1}$ and $\beta_{\mathrm{r}}$ are the beacon opening angles of the left and right side walls, respectively. The corresponding angles $\alpha_{11}$ and $\alpha_{1 \mathrm{r}}$ are equal to $\alpha_{1}: \alpha_{1 \mathrm{l}}=\alpha_{1 \mathrm{r}}=\alpha_{1}$. 

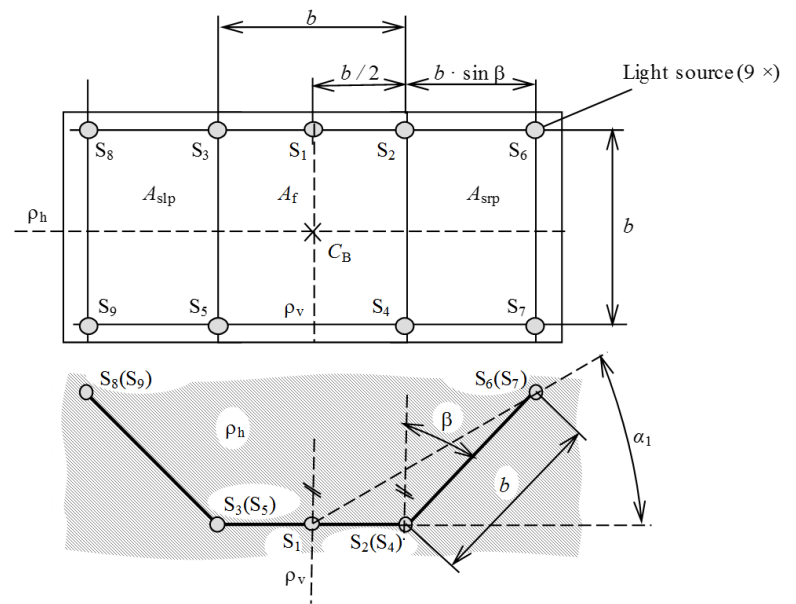

Fig. 1. Optical beacon layout chart.

Passive camera measurements can be expressed by two mathematical models. The first model consists of analytical relations for the separate measurement of the beacon range and camera azimuth, see Part II-A. The second model is a set of analytical relations that are used for the simultaneous measurement of the range and azimuth, see II-B.

\section{A. The separate measurement of range and azimuth}

In the case of separate measurement, the beacon range and the camera azimuth are determined by separate relations. The calculation of the azimuth is either independent of the range or it is conditioned by knowledge of the range. The explicit relation for beacon range $R(\mathrm{~m})$ is as follows [13]:

$$
R=f \cdot\left(\frac{b}{b^{\prime}}-1\right)
$$

where $b$ is the base length, $b^{\prime}$ is the base image length, $(\mathrm{m})$; $f$ is the focal length of the camera lens, $(\mathrm{m})$.

In order to determine the base image length $b^{\prime}$, the distance between the images of the diodes lying above one another must be used. Their connecting lines are parallel to the plane of symmetry; e.g. diodes $\mathrm{S}_{8}, \mathrm{~S}_{9}$ and $\mathrm{S}_{3}, \mathrm{~S}_{5}$ etc. The distance $b^{\prime}$ between the images of these diodes does not change with the azimuth. It is determined by the pixel size and a number of the relevant pixels.

Azimuth can be measured using the ratio of the distances between the images of corresponding diodes, for example $S_{2}$, $\mathrm{S}_{6}$ and $\mathrm{S}_{8}, \mathrm{~S}_{3}$. This ratio is given by [13]:

$$
\frac{b_{26}^{\prime}}{b_{83}^{\prime}}=\frac{\sin \beta \cdot \cos \omega+\cos \beta \cdot \sin \omega}{\sin \beta \cdot \cos \omega-\cos \beta \cdot \sin \omega},
$$

where $b_{26}^{\prime}$ and $b_{83}^{\prime}$ are the distances between the images of the diodes $S_{2}, S_{6}$ and $S_{8}, S_{3}$, respectively, in the area of the camera detector, $(\mathrm{m}) ; \omega$ is the azimuth, $\left(^{\circ}\right)$.

The equation 5 is derived for orthographic projections of the diodes onto a plane perpendicular to the optical axis of the camera. It does not take into account the issue of perspective when projecting spatial objects onto a plane [14]. As a result, it can be used with sufficient accuracy only at relatively large distances.

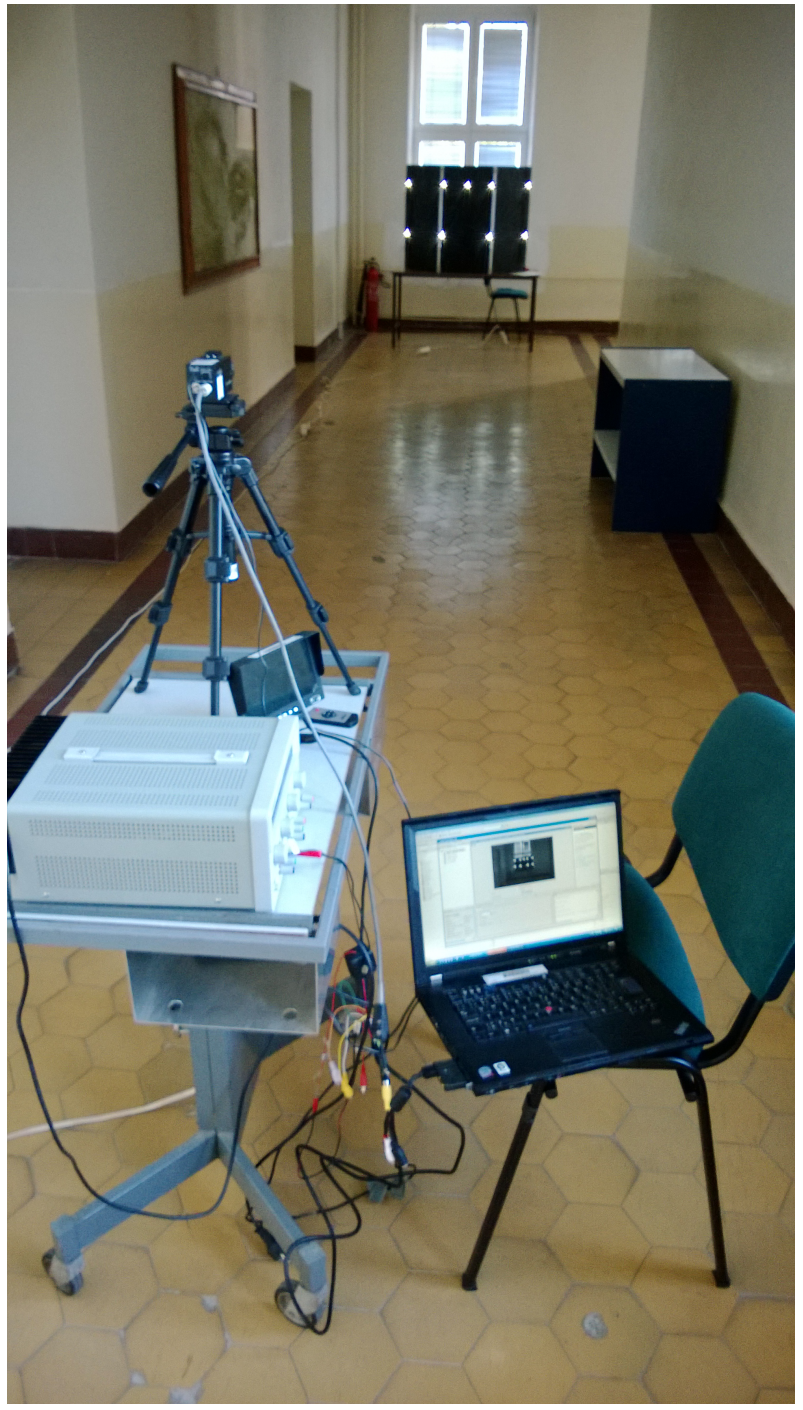

Fig. 2. Experiment arrangement.

The angle $\omega$ cannot be expressed explicitly and it is, therefore, determined numerically. The measurement of the azimuth is independent of the beacon range.

The span of measurable ranges spreads toward smaller values using a relation that takes into account the perspective projection [13], [14]:

$$
\frac{b_{26}^{\prime}}{b_{83}^{\prime}}=\frac{\frac{d_{16} \cdot \cos \left(\alpha_{1}+\omega\right)}{R+d_{16} \cdot \sin \left(\alpha_{1}+\omega\right)}-\frac{d_{12} \cdot \cos \omega}{R+d_{12} \cdot \sin \omega}}{\frac{d_{16} \cdot \cos \left(\alpha_{1}-\omega\right)}{R+d_{16} \cdot \sin \left(\alpha_{1}-\omega\right)}-\frac{d_{12} \cdot \cos \omega}{R-d_{12} \cdot \sin \omega}} .
$$

Even in this case, the angle $\omega$ must be determined numerically. Since (6) includes the beacon range $R$, the range must be calculated preliminarily from (4).

\section{B. Simultaneous measurement of the range and azimuth}

The principle of simultaneous measurement of the beacon range and the camera azimuth is based on the numerical calculation of the mean of the beacon ranges determined from the distances between images of the relevant diodes 
constituting a measuring pair. The range mean is then used to determine the root mean square (rms) of the range differences. The range difference is the difference between the range mean and the range calculated for a given measuring pair of diodes.

Supposing that the $d_{81}=d_{16}$ and $\alpha_{11}=\alpha_{1 \mathrm{r}}$ equations are valid, the beacon ranges calculated for individual diode pairs are as follows:

$$
\begin{gathered}
R_{31}=f \cdot\left[\frac{b \cdot \cos (-\omega)}{2 \cdot b_{31}^{\prime}}-1\right]-\frac{b}{2} \cdot \sin (-\omega), \\
R_{12}=f \cdot\left(\frac{b \cdot \cos \omega}{2 \cdot b_{12}^{\prime}}-1\right)-\frac{b}{2} \cdot \sin \omega, \\
R_{81}=f \cdot\left[\frac{d_{16} \cdot \cos \left(\alpha_{1}-\omega\right)}{b_{81}^{\prime}}-1\right]-d_{16} \cdot \sin \left(\alpha_{1}-\omega\right), \\
R_{16}=f \cdot\left[\frac{d_{16} \cdot \cos \left(\alpha_{1}+\omega\right)}{b_{16}^{\prime}}-1\right]-d_{16} \cdot \sin \left(\alpha_{1}+\omega\right),
\end{gathered}
$$

where $b_{31}^{\prime}, b_{13}^{\prime}, b_{81}^{\prime}, b_{16}^{\prime}$ are distances between the image of the $\mathrm{S} 1$ diode and the images of the $\mathrm{S}_{3}, \mathrm{~S}_{2}, \mathrm{~S}_{8}, \mathrm{~S}_{6}$ diodes, respectively, in the plane of the camera detector, $(\mathrm{m})$; $R_{3} 1 \ldots R_{16}$ are the beacon ranges determined on the basis of the distances between the images of the relevant diodes.

In approximation of the geometrical optics, (7) to (10) were derived using the image position and magnification formulas of the lens and the dependency of the distance between the relevant diode and optical axis on the range $R$ and azimuth $\omega$. The perspective projection is taken into consideration as well.

Fig. 3 illustrates the principle of the method. The diodes $\mathrm{S}_{1}, \ldots \mathrm{S}_{8}$, and $\mathrm{S}_{1 \omega}, \ldots \mathrm{S}_{8 \omega}$ represent the beacon position for camera azimuth $\omega=0^{\circ}$, and $\omega \neq 0^{\circ}$, respectively. The plane $\rho_{\omega 0}$ is the reference plane. It is determined by the front wall position for $\omega=0^{\circ}$. Mutual distances between diode images, here $S_{1 \omega}^{\prime}, S_{2 \omega}^{\prime}$ and $S_{8 \omega}^{\prime}$ only, change with $R$ and $\omega$.

The corresponding dependence on $\omega$ is illustrated in Fig. 4. The individual pictures are for the beacon range $R=40.96 \mathrm{~m}$, and camera azimuth $\omega=\{2 ; 37\}^{\circ}$. These pictures demonstrate the shift of the diode images for different values of the azimuth.

The mentioned mean of the calculated ranges $\bar{R}(\mathrm{~m})$ is expressed by the following formula:

$$
\bar{R}=\frac{R_{31}+R_{12}+R_{81}+R_{16}}{4} .
$$

The range difference $D_{\mathrm{ij}}(\mathrm{m})$ for the $i, j$ diode pair is given by the formula:

$$
D_{\mathrm{ij}}=R_{\mathrm{ij}}-\bar{R} \text {. }
$$

The rms of the range differences $D_{\text {rms }}(\mathrm{m})$ is as follows

$$
D_{\mathrm{rms}}=\sqrt{\frac{1}{4}\left(D_{31}^{2}+D_{12}^{2}+D_{81}^{2}+D_{16}^{2}\right)} .
$$

Changing the azimuth in (7) to (10) leads to changes in the mean of the calculated ranges and the rms of the range differences. Assuming that the input azimuth is equal to the true azimuth, the beacon parameters are set exactly according to the selected values, and the camera has a high resolution,

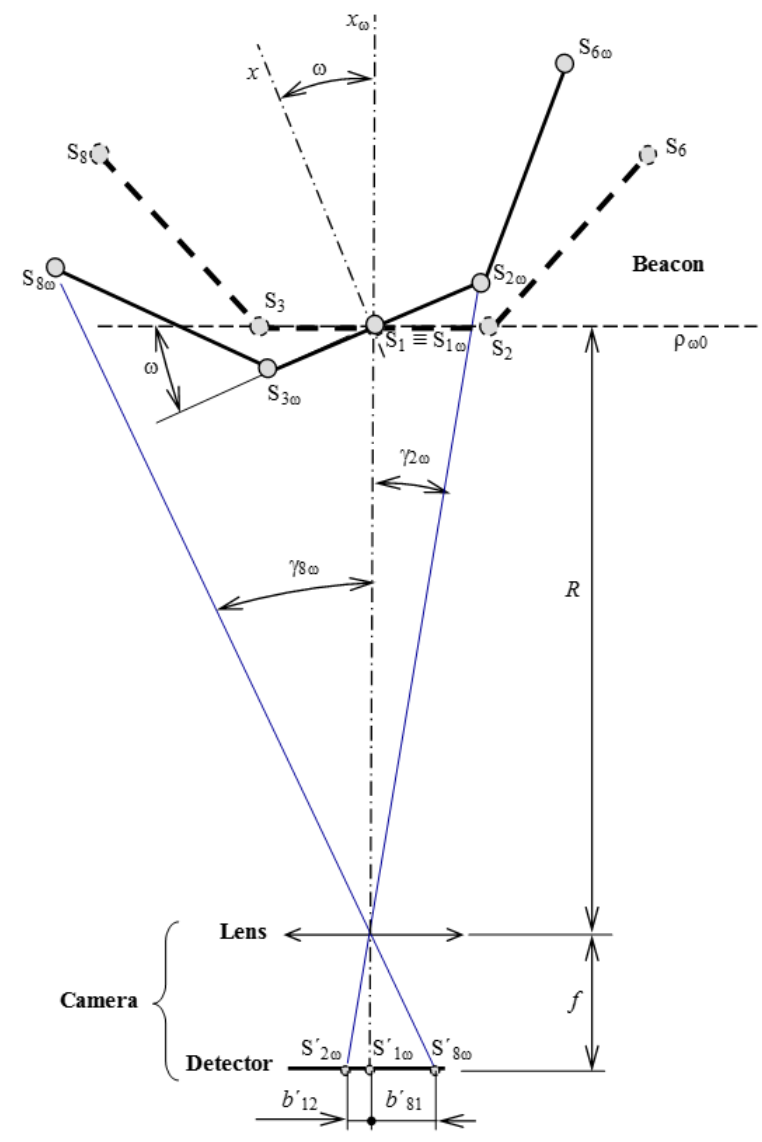

Fig. 3. Scheme of the measuring system.

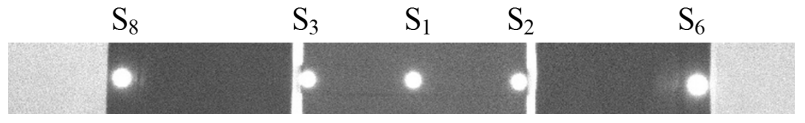

(a)

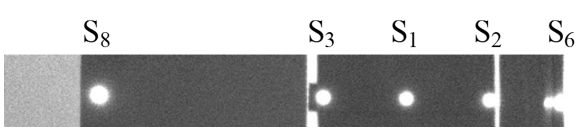

(b)

Fig. 4. View of the diodes in the beacon upper line: $R=40.96 \mathrm{~m}$, (a) $\omega=2^{\circ}$, (b) $\omega=37^{\circ}$.

we could theoretically expect the rms of the range differences to be practically zero.

Because of measurement errors, the sought azimuth is such (substituted into (7p) to (10), for which the rms of the range differences is minimal. In this case, we consider the mean of the calculated ranges to be the sought distance between the beacon and the camera.

\section{Measurement Results}

This section presents the results of the indicative and subsequent experiments following the model for the simultaneous measurement of range and azimuth. The aim of the indicative experiment was to assess whether the proposed method is applicable. It was conducted using a Mintron OS-45 D camera 
TABLE I

MEASURED VALUES OF (A) AZIMUTH AND (B) BEACON RANGE.

(A)

\begin{tabular}{|c|c|c|c|c|}
\hline \multirow{4}{*}{$\begin{array}{l}\text { Actual Beacon } \\
\text { Range (m) }\end{array}$} & \multicolumn{4}{|c|}{ Lens Focal Length (mm) } \\
\hline & 8 & 16 & 25 & 120 \\
\hline & \multirow{2}{*}{\multicolumn{4}{|c|}{$\begin{array}{c}\text { Supposed Azimuth } \omega=0^{\circ} \\
\text { Measured Azimuth }\left(^{\circ}\right)\end{array}$}} \\
\hline & & & & \\
\hline 14.58 & 0.70 & 0.70 & 0 & $\times$ \\
\hline 26.19 & 0 & 2.54 & 1.68 & 0.52 \\
\hline \multirow[t]{3}{*}{47.24} & 0 & 0 & 0 & 0.62 \\
\hline & \multicolumn{4}{|c|}{ Supposed Azimuth $\omega=15^{\circ}$} \\
\hline & \multicolumn{4}{|c|}{ Measured Azimuth $\left({ }^{\circ}\right) /$ Percentage Error $(\%)$} \\
\hline 14.58 & $15.58 / 3.87$ & $15.52 / 3.47$ & $15.62 / 4.13$ & $\times$ \\
\hline 26.19 & $13.08 /-12.80$ & $14.35 /-4.33$ & $14.87 /-0.87$ & $14.87 /-0.87$ \\
\hline \multirow[t]{3}{*}{47.24} & $13.65 /-9.00$ & $11.66 /-22.30$ & $15.11 / 0.73$ & $14.51 /-3.27$ \\
\hline & \multicolumn{4}{|c|}{ Supposed Azimuth $\omega=\mathbf{3 0}^{\circ}$} \\
\hline & \multicolumn{4}{|c|}{ Measured Azimuth $\left({ }^{\circ}\right) /$ Percentage Error $(\%)$} \\
\hline 14.58 & $28.82 /-3.93$ & $29.32 /-2.27$ & $28.88 /-3.73$ & $\times$ \\
\hline 26.19 & $29.87 /-0.43$ & $29.65 /-1.17$ & $29.6 /-1.33$ & $29.36 /-2.13$ \\
\hline \multirow[t]{3}{*}{47.24} & $x$ & $30.98 / 3.27$ & $29.83 /-0.57$ & $30.43 / 1.43$ \\
\hline & \multicolumn{4}{|c|}{ (B) } \\
\hline & \multicolumn{4}{|c|}{ Lens Focal Length (mm) } \\
\hline \multirow{3}{*}{$\begin{array}{l}\text { Actual Beacon } \\
\text { Range (m) }\end{array}$} & 8 & 16 & 25 & $120 \mathrm{~g}$ \\
\hline & \multicolumn{4}{|c|}{ Supposed Azimuth $\omega=0^{\circ}$} \\
\hline & \multicolumn{4}{|c|}{ Measured Range (m) / Percentage Error $(\%)$} \\
\hline 14.58 & $13.69 /-6.10$ & $13.83 /-5.14$ & $13.97 /-4.18$ & $\times$ \\
\hline 26.19 & $24.62 /-5.99$ & $24.85 /-5.11$ & $24.90 /-4.93$ & $25.51 /-2.60$ \\
\hline \multirow[t]{3}{*}{47.24} & $44.95 /-4.85$ & $44.15 /-6.54$ & $44.93 /-4.89$ & $45.55 /-3.58$ \\
\hline & \multicolumn{4}{|c|}{ Supposed Azimuth $\omega=15^{\circ}$} \\
\hline & \multicolumn{4}{|c|}{ Measured range (m) / Percentage error $(\%)$} \\
\hline 14.58 & $13.86 /-4.93$ & $14.00 /-3.98$ & $14.15 /-2.95$ & $\times$ \\
\hline 26.19 & $24.62 /-5.99$ & $25.04 /-4.39$ & $25.20 /-3.78$ & $25.52 /-2.56$ \\
\hline \multirow[t]{3}{*}{47.24} & $44.70 /-5.38$ & $47.22 /-0.04$ & $45.00 /-4.74$ & $45.06 /-4.61$ \\
\hline & \multicolumn{4}{|c|}{ Supposed Azimuth $\omega=30^{\circ}$} \\
\hline & \multicolumn{4}{|c|}{ Measured range (m) / Percentage error $(\%)$} \\
\hline 14.58 & $14.03 /-3.77$ & $14.06 /-3.56$ & $14.25 /-2.26$ & $x$ \\
\hline 26.19 & $25.01 /-4.51$ & $25.00 /-4.54$ & $25.33 /-3.28$ & $26.73 / 2.06$ \\
\hline 47.24 & $x$ & $45.03 /-4.68$ & $45.71 /-3.24$ & $45.30 /-4.11$ \\
\hline
\end{tabular}

and four different lenses. Their focal lengths were $\{8 ; 16 ; 25$; $120\} \mathrm{mm}$. The beacon base length was $47 \mathrm{~cm}$ and the beacon opening angle was $47^{\circ}$. The measurement was performed for three azimuth values and three range values for every lens: $\omega=\{0 ; 15 ; 30\}^{\circ}, R=\{14.58 ; 26.19 ; 47.24\} \mathrm{m}$, see Table I For each configuration, the beacon position was measured only once.

Table $\square$ is divided into two sections. The first one contains the found azimuth values, as well as the corresponding percentage errors, for $\omega=\{15 ; 30\}^{\circ}$. In the second section, the values of the beacon ranges and the corresponding percentage errors for all measured ranges are presented.

Cells containing a cross indicate such configurations of the focal length, beacon range and camera azimuth which did not allow the evaluation of the acquired snapshot and the calculation of an appropriate quantity. In the case of the 8 $\mathrm{mm}$ lens, the reason was low resolution. The images of the diodes lying next to each other $\left(\mathrm{S}_{8}, \mathrm{~S}_{3}\right.$ or $\left.\mathrm{S}_{6}, \mathrm{~S}_{2}\right)$ overlapped. When using the $120 \mathrm{~mm}$ lens with a large magnification and a small field of view, only a part of the beacon was displayed.

The errors were mainly caused by the fact that the actual values of the beacon parameters (base and opening angle) differed from the supposed values because the beacon was not firm enough. In the case of the azimuth, the absolute values of most percentage errors did not exceed the level of $5 \%$. This error was extreme $(22.30 \%)$ in one case. The probable cause may be a gross error when reading the coordinates of the diode images. In the case of the range, no absolute value of the percentage error value exceeded the $7 \%$ level. The mean 
TABLE II

ABSOLUTE PERCENTAGE OF THE BEACON RANGE.

\begin{tabular}{|c|c|c|c|c|c|c|c|c|c|c|c|c|}
\hline \multirow{5}{*}{$\begin{array}{l}\text { Actual beacon } \\
\text { range (m) }\end{array}$} & \multicolumn{12}{|c|}{ Number of measurement } \\
\hline & \multicolumn{4}{|c|}{$1^{\text {st }}$} & \multicolumn{4}{|c|}{$\mathbf{3}^{\text {rd }}$} & \multicolumn{4}{|c|}{$5^{\text {th }}$} \\
\hline & \multicolumn{4}{|c|}{ Camera angle $\left(^{\circ}\right)$} & \multirow{2}{*}{\multicolumn{4}{|c|}{$\begin{array}{l}\text { Camera angle }\left({ }^{\circ}\right) \\
\text { Absolute percentage error }(\%)\end{array}$}} & \multirow{2}{*}{\multicolumn{4}{|c|}{$\begin{array}{c}\text { Camera angle }\left({ }^{\circ}\right) \\
\text { Absolute percentage error }(\%)\end{array}$}} \\
\hline & Absc & e perc & age er & $(\%)$ & & & & & & & & \\
\hline & $\omega_{\mathrm{g} 1}$ & $\omega_{\mathrm{g} 2}$ & $\omega_{\mathrm{g} 3}$ & $\omega_{\mathrm{g} 4}$ & $\omega_{\mathrm{g} 1}$ & $\omega_{\mathrm{g} 2}$ & $\omega_{\mathrm{g} 3}$ & $\omega_{\mathrm{g} 4}$ & $\omega_{\mathrm{g} 1}$ & $\omega_{\mathrm{g} 2}$ & $\omega_{\mathrm{g} 3}$ & $\omega_{\mathrm{g} 4}$ \\
\hline \multirow{2}{*}{32.28} & 0 & 15 & 30 & 39 & 0 & 15 & 30 & 39 & 0 & 15 & 30 & 39 \\
\hline & 1.22 & 0.52 & 0.04 & 0.01 & 1.50 & 0.93 & 0.26 & 0.41 & 1.50 & 1.13 & 0.52 & 0.11 \\
\hline \multirow{2}{*}{$\begin{array}{c}33.13 \\
\text { (1st series) }\end{array}$} & 1 & 14 & 29 & 38 & 1 & 14 & 29 & 38 & 1 & 14 & 29 & 38 \\
\hline & 1.52 & 0.86 & 0.52 & 0.82 & 1.14 & 0.78 & 0.01 & 0.55 & 1.14 & 0.74 & 0.26 & 0.72 \\
\hline \multirow{2}{*}{$\begin{array}{c}33.13 \\
\text { (2nd series) }\end{array}$} & 2 & 13 & 28 & 37 & 2 & 13 & 28 & 37 & 2 & 13 & 28 & 37 \\
\hline & 0.75 & 0.99 & 1.10 & 0.09 & 1.26 & 0.71 & 0.10 & 0.01 & 1.17 & 1.04 & 0.52 & 0.08 \\
\hline \multirow{2}{*}{39.47} & 0 & 15 & 30 & 39 & 0 & 15 & 30 & 39 & 0 & 15 & 30 & 39 \\
\hline & 1.05 & 1.10 & 0,18 & 0,18 & 1,15 & 0,96 & 0,09 & 0,54 & 1,15 & 0,75 & 0,23 & 0,42 \\
\hline \multirow{2}{*}{39.73} & 1 & 14 & 29 & 38 & 1 & 14 & 29 & 38 & 1 & 14 & 29 & 38 \\
\hline & 1.03 & 0.95 & 0.72 & 0.21 & 1.03 & 0.68 & 0.42 & 0.31 & 1.03 & 0.97 & $\times$ & 0.44 \\
\hline \multirow{2}{*}{40.96} & 2 & 13 & 28 & 37 & 2 & 13 & 28 & 37 & 2 & 13 & 28 & 37 \\
\hline & 0.90 & 0.94 & 0.56 & 0.03 & 1.10 & 0.93 & 0.04 & 0.23 & 1.10 & 0.73 & 0.19 & 0.03 \\
\hline \multirow{2}{*}{54.76} & 0 & 15 & 30 & 39 & 0 & 15 & 30 & 39 & 0 & 15 & 30 & 39 \\
\hline & 0.73 & 0.94 & 0.60 & 0.86 & 0.73 & 0.65 & 0.37 & 1.17 & 0.73 & 0.46 & 0.60 & 0.86 \\
\hline
\end{tabular}

TABLE III

MEAN AND SAMPLE STANDARD DEVIATION OF THE BEACON RANGE.

\begin{tabular}{|c|c|c|c|c|c|c|c|}
\hline $\begin{array}{l}\text { Actual beacon } \\
\text { range (m) }\end{array}$ & 32.28 & 33.13 & 33.13 & 39.47 & 39.73 & 40.96 & 54.76 \\
\hline $\begin{array}{l}\text { Mean of the } \\
\text { beacon range (m) }\end{array}$ & 32.04 & 32.98 & 32.91 & 39.28 & 39.49 & 40.74 & 54.30 \\
\hline $\begin{array}{l}\text { Standard } \\
\text { deviation }(\mathbf{m})\end{array}$ & 0.23 & 0.23 & 0.18 & 0.24 & 0.19 & 0.20 & 0.21 \\
\hline
\end{tabular}

absolute percentage errors determined from all the measured values were $3.18 \%$ for the azimuth and $4.17 \%$ for the range. The error values listed in Table $\mathrm{I}$ are not results of the proper analysis of the accuracy. They indicate whether the method is usable. But stated values of the simultaneous method and the errors of the neural network method [10], [11] are of the same order.

During the second experiment, 140 pictures, which were used for the determination of the measured quantities, were recorded. All images were acquired with the camera MOTICAM 1080. The focal length of the objective was $120 \mathrm{~mm}$. The camera image detector had the aspect ratio of 16:9, the diagonal of $6.4 \mathrm{~mm}$, and the pixel number of $1920 \times 1080$. The beacon was placed on the Thorlabs RBB12A rotation stage which enabled to set the actual camera azimuth with the accuracy of 5 minutes of arc. The actual beacon range was measured with the Leica Disto D510 laser distance meter. The instrument had the accuracy of $1 \mathrm{~mm}$.

The mathematical model was more specified. The real values of the parameters $d_{16}$ and $\alpha_{1}$ were substituted into (4) to (7). They differed for individual side walls. It emerged that these parameters must be determined very exactly because deviations between their actual values of the beacon and the values used in the mathematical model caused the serious measurement errors.

Another important component of the measurement system is the focal length. The deviations between its actual camera value and the value substituted into mathematical model need not necessarily equal zero. For the different beacon ranges and the constant focal length of the model, it was verified that the percentage errors of the measured beacon range changed in a small span. If another focal length was used, these errors shifted by the values which were approximately the same for the various ranges. It follows that the calibration of the measuring system can be easily made, if necessary, by means of an additive coefficient which modifies the value of the objective focal length in the mathematical model. What is considered as important is the fact that the influence of the focal length discrepancies on the accuracy of the camera azimuth measurement is negligible.

What can cause the additional measurement errors is a manual way of the evaluation of diode positions in the picture. The center of each diode is evaluated by an observer. As a result, due to a pixel size and a diode shape in the picture it is difficult to find the true diode center. The error of the diode position determination could be pm4 pixels or less for 
a chosen resolution. It depends on the pixel size of the diode in the picture and its position on the beacon. It is more difficult to evaluate a position of the diodes on the side walls especially for higher values of $\omega$. Moreover, in some cases, the reflection of diode light from the beacon surface create secondary false sources that can interfere with the observer and consequently cause inaccurate reading of the diode positions, see Fig. 4 With the aim to estimate the influence of the above mentioned errors the pixel numbers of the individual diodes were changed one by one. These changes were made for the combinations of two diodes as well. The results of the first measurement of the second experiment for the range of $32.28 \mathrm{~m}$ and the azimuth of $15^{\circ}$ were used. In comparison with the original results, the maximum percentage and absolute deviations in the range and azimuth were $0.36 \%$ and $0.55^{\circ}$, respectively.

The second experiment was performed for four groups of the camera azimuth values and six beacon range values: $\omega_{\mathrm{g} 1}=\{0 ; 1 ; 2\}^{\circ}, \omega_{\mathrm{g} 2}=\{13 ; 14 ; 15\}^{\circ}$, $\omega_{\mathrm{g} 3}=\{28 ; 29 ; 30\}^{\circ}, \omega_{\mathrm{g} 4}=\{27 ; 28 ; 29\}^{\circ}$, $R=\{32.28 ; 33.13 ; 39.47 ; 39.73 ; 40.96 ; 54.76\}$ m. Every range with one value chosen from every group of angles was measured five times. Two series of the measurement were made for the range of $33.13 \mathrm{~m}$. If the focal length of $120 \mathrm{~mm}$ was inserted into the mathematical model, the range errors were around $5 \%$. That was unacceptable as it was too much above than expected. Therefore $125 \mathrm{~mm}$ focal length was used for the mathematical model.

The gathered data were used for the computation of some statistical variables. For three (1st, 3rd, 5th) measurements, the absolute percentage errors of the range are listed in Table II The cross in the table indicates the gross error. The mean absolute percentage errors of the range $M A P E_{\mathrm{R}}(\%)$ in the dependence on the azimuth are depicted in Fig. 5 for every set range. The black curve is for the range of $54.76 \mathrm{~m}$. Means $\bar{R}$ and sample standard deviations $s_{\mathrm{R}}$ of the range are listed in Table III

The mean errors $M E_{\omega}\left({ }^{\circ}\right)$ and the mean absolute errors $M A E_{\omega}\left({ }^{\circ}\right)$ of the azimuth are depicted in Fig. 6 and Fig. 7 for all ranges as well. The gray curves are for the ranges of $\{32.28$; $39.47 ; 39.73 ; 40.96\} \mathrm{m}$ and $33.13 \mathrm{~m}$ of the second series. The black and red curves are for $54.76 \mathrm{~m}$ and for $33.13 \mathrm{~m}$ of the first series, respectively. The individual values of the variables were computed from the data which had been acquired from all five measurements.

The obtained data are slightly optimistic. The maximum absolute percentage error and sample standard deviation of the range are $1.52 \%$ and $0.24 \mathrm{~m}$, respectively. The results for the azimuth are slightly worse but satisfactory. The absolute error values of $(-0.1 ; 0.1)^{\circ}$ are $20 \%$ of the all sample number. They are $58.6 \%$ for the interval $(-0.3 ; 0.3)^{\circ}$ and $72.9 \%$ for the interval $(-0.5 ; 0.5)^{\circ}$. The biggest absolute errors were $1.64^{\circ}$ for the range of $54.76 \mathrm{~m}$ and azimuth of $39^{\circ}$, and $-0.89^{\circ}$ for the range of $33.13 \mathrm{~m}$ and azimuth of $39^{\circ}$.

\section{CONCLUSION}

The proposed passive method of the simultaneous measurement of the relative position of the optical beacon is based

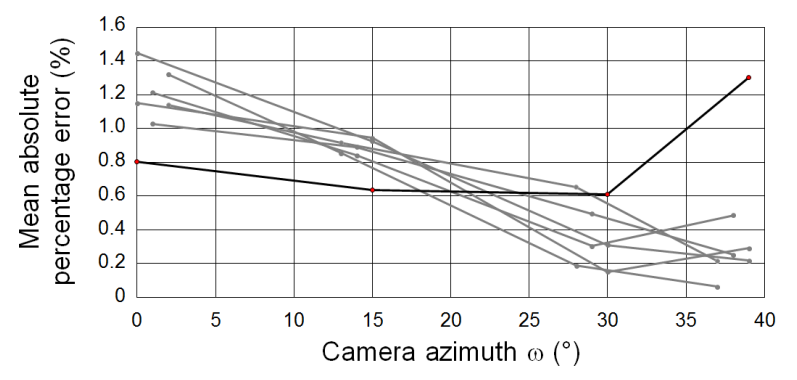

Fig. 5. Mean absolute percentage error of the range.

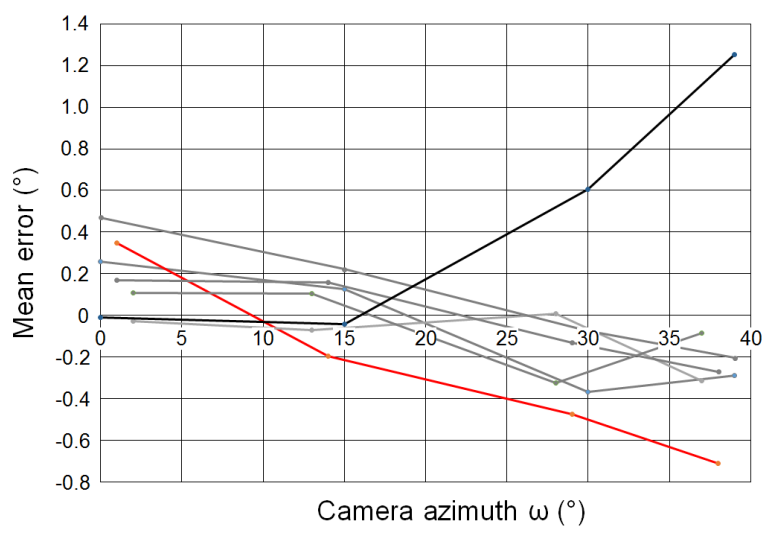

Fig. 6. Mean error of the azimuth

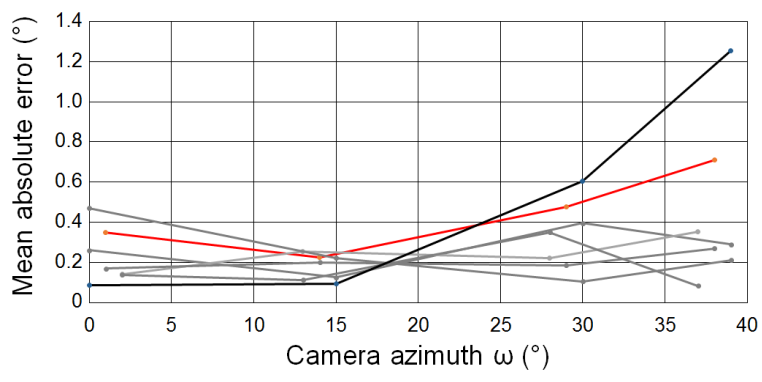

Fig. 7. Mean absolute error of the azimuth.

on the use of a defined spatial arrangement of diodes forming an optical beacon. By evaluating the corresponding distances between individual diode images in the camera sensor plane, the beacon range and potentially both the camera azimuth and the elevation relative to the beacon can be determined. The experimental beacon and the presented mathematical model allow measuring the range and azimuth only. The maximum measurable range is determined by the camera resolution, which is, for the given sensor, determined by the lens focal length. The minimum measurable range depends on the beacon size and the camera field of view. All beacon diodes must be displayed on the detector sensitive surface. The theoretical azimuth measurement span is given by the beacon opening angle: $\omega \in(-\beta, \beta)$.

Table I presents the results of the experimental measurement of the beacon range and camera azimuth. These results are only indicative as the declared actual beacon parameters and azimuth values could not be fully met. 
If the true base and beacon opening angle differ from the values inserted into mathematical model, methodological errors arise. The instrument errors depend on the camera resolution and method of image processing. Other errors result from mathematical models, approximations, influence of elevation angle, lens distortion and lens inaccuracy. The equations (7) to (10) are not valid for the elevation angle which differs from zero. However, during the experiment, its possible random values were small, and its effect on the accuracy was small as well. The lens quality influences the method precision significantly. The lens distortions were verified but they were not measurable. Other lens inaccuracies were compensated manually.

As expected, the results of the second experiment are better than the results of the first one, see Table [II. Table III and Fig. 5 to Fig. 7. A camera with the higher resolution was used, parameters of the beacon were adjusted more exactly, and both the actual beacon range and azimuth angle were set more precisely in the comparison with the first experiment.

This experiment was particularly aimed at avoiding the imperfections which caused methodological errors. This requirement concerns the basic beacon parameters primarily. Nevertheless, these parameters for the individual side walls can differ but their actual values must be known precisely. These values must be inserted into mathematical model. In the case of the beacon range, the measuring system can be easily calibrated by changing the lens focal length of the mathematical model.

The measurement of the object position with the presented method can be potentially applied during day and night landings of helicopters using TV or thermal imaging cameras. Infrared cameras can work with a beacon made of natural infrared radiation sources. These sources can be created, for example, by applying a contrast material to defined locations of a plate with a homogeneous surface.

\section{REFERENCES}

[1] A. Bourdonnaye, R. Doskocil, V. Krivanek, and A. Stefek, "Practical Experience with Distance Measurement Based on the Single Visual Camera," Advances in Military Technology, vol. 8, no. 1, pp. 51-58, 2012.

[2] R. Doskocil, J. Fischer, V. Krivanek, and A. Stefek, "Measurement of Distance by Single Visual Camera at Robot Sensor Systems," in Proc. 15th Mechatronika 2012, Praha, pp. 143-149, 2012.

[3] M. T. Bui, R. Doskocil, V. Krivanek, T. H. Ha, Y. T. Bergeon, and P. Kutilek, "Indirect Method to Estimate Distance Measurement Based on Single Visual Cameras," in International Conference on Military Technologies, Brno, pp. 695-700, 2017.

[4] H. Kondo et al., "Passive acoustic and optical guidance for underwater vehicles," in 2012 Oceans - Yeosu. doi: 10.1109/OCEANSYeosu.2012.6263572, 2012.

[5] M. T. Bui, R. Doskocil, and V. Krivanek, "Distance and Angle Measurement Using Monocular Vision," in Proceedings of the 2018 18th International Conference on Mechatronics, Brno, pp. 422-427, 2018

[6] M. Ramezani and K. Khoshelham, "Vehicle positioning in GNSS deprived urban areas by stereo visual-inertial odometry," IEEE Transactions on Intelligent Vehicles, vol. 3, no. 2, pp. 208-217, 2018.

[7] Y. Mizuchi, T. Ogura, Y. B. Kim, et al., "Accuracy evaluation of camerabased position and heading measurement system for vessel positioning at a very close distance,'in 15th International Conference on Control, Automation and Systems (ICCAS), Busan, pp. 747-751, 2015.

[8] M. Wang, Y. Liu, D. Su, et al., "Accurate and real-time 3D tracking for the following robots by fusing vision and ultra-sonar information, "IEEE/ASME Transactions on Mechatronics, vol. 23, no. 3, pp. 997 1006,2018
[9] T. Saito, K. Nomura, Y. Yamazaki, et al., "Position measurement for a mobile weed mowing robot by a camera and a laser rangefinder," in International Symposium on Micro-NanoMechatronics and Human Science (MHS), Nagoya, pp. 1-5, 2017.

[10] M. Polasek, "Using a neural network to determine the position of a moving object," [in Czech] in Measurement, diagnostics, reliability of aircraft onboard systems, Brno, pp. 242-250, 2017.

[11] M. Polasek, V. Pucek, J. Nemecek, and R. Bloudicek, "Determining the Position Using Neural Network," in Proc. 22nd International Scientific Conference. Transport means, Kaunas, pp. 286-289, 2018.

[12] J. Nemecek and M. Polasek, "Measurement of Relative Position of Camera and Optical Beacon by Simultaneous Passive Method," in 41st International Conference on Telecommunications and Signal Processing (TSP), Athens, pp. 56-59, 2018.

[13] J. Nemecek, "Passive Measurement of Camera Position Relative to Optical Beacon," [in Czech] in Measurement, diagnostics, reliability of aircraft onboard systems, Brno, pp. 218-226, 2017.

[14] Kjell J. Gasvik, Optical Metrology, Chichester: John Wiley \& Sons, 2002 . 